Pistolenknall, Blasen, Luftzug usw., hervorbringen. Die zweite Erscheinung der Teilchenzerstreuting ist nicht isoliest zu erhalten, kann aber noch deutlicher gemacht werden, wenn die Gefaßwande einander noch mehr genähert sind, weil dadurch die Reibung der gesamten Luftnasse erhöht wird. Die Zerstreuung der Teilchen ist un so großBer, je gröller die Energie der Entladung ist. Für denselben Kondensator und dieselbe Funkenlănge ist sie bei gegebener Fnergie um so größer, je kleiner die Selbstinduktion, d. h. je rascher die Schwingungsperiode ist. Die Schwingungen variierten bei difsen Versuchen $z$ wischen 250000 und einigen zehn Millionen in der Sekunde.

E. $M$.

Henri, V., und Lifchitz, S., Kinematographische Untersuchung der durch sehr rasche Schallstobe hervorgerufenen Verschiebung ultra. mikroskopischer Teilcilen. (Compt. rend 152, 953-955, 1911.)

Im AnschlúB an die vorstehend referierte Arbeit untersuchen dte Verfasser die Erscheinung kinematographisch. Es zeigt sich, $\mathrm{daB}$ die Teilchenverschiebung durch Lufterschitterung erzeugt wird, also eine mechanische Wirkung ist. Bringt man zwischen Funkenerzeuger und Beobachtungsgefab etwa ein Glasblättchen oder Quarz- oder Papierblatt, so unterbleibt dic Erscheinung. Sie ist auch von der elektrischen Ladung der Teilchen unabhängig, denn sle tritt sowohl bei Tabakrauch au, dessen Teilchen im allgemeinen negativ sifh, wie bei den elektrisch neutralen Teilchen des Salmiakdampies. Weiter ist bemerkenswert, daB Teilchen, die nur $20-30 \mu$ voneinander entfern! sind, unter dem Einfluß sehr rascher Luftsioße häufig in entgegengesetzter Richtung verschoben werden. Diese Verschijebungen siud rascher und grober, als die bei der Brown'schen Bewegung. Das dentet auf Luftwirbel von sehr kleinem Radius. Die Vcischiebung der Teilchen bei der Brown'schen Bewegung betragt $5--6 \mu$ in der Sekunde, die durch die Schallstôße hervorgerufenen dagegen schatzungsweise $1 \mathrm{~mm}$ in der Sekunde. E. M.

Lepkowsky, W. von, Kritische Erscheinungen in Losungen unter dem Kardioid-Ultramikroskop. (Zeltschr. I. physik. Chcm. 75, 608-614, 1910)

Der Verfasser hat Lösungen unter dem Kardıoidultramikroskop bis zur kritischen Temperatur und darunter abgekuhit i"d dann wieder bis iber diese Temperatur erwärmt. Es zeigte sich dabei, daß der mittlere Teil des Gesichtsfeldes, bei Annäherung an den kritischen Punkt von then, immer beller wird, dann ein starkes Flimmern, mit weiterer Abkühlung endlich Submikronen erkentict läbt, die sich tnit großer Geschwindigkeit bewegren und aneinander stoßen, bis schlieblich verhältnismaibig grobe Tropfen, oft auch Tropfenkonglomerate, erscheinen. In diesem Momente ist die kritische Temperatur und die Entmischung erreicht. Beim langsamen Erwärmen sind die umgekehrten Erscheinungen wahrzunehmen. Im kritischen Punkte ist eigentlich die Grenzflächenspannung zwischen beiden Phasen und ebenso die Differenz der Brechungsexponenten Null. Das scheint aber für die kleinen Tröpt shen nicht zuzutreffen. Dab dic- Diffusion in soichen kritischen Lösungen eine selir kleine Geschwindigkeit besitct. gibt daraus hervor, daB die Tropfchen bei mehrmaligu Wiedererwarmung und Athkuhlung stets an derselben Stelle auftreten. Die relative Verteilung der beiden Substanzen gegeneinander wird nicht wesentlich geändert
Liesegang, R. Ed., Ueber die Reifung von Silberhaloidemulsionen. (Zeitschr, f. physik. Chem.75, $374-377,1910$.)

Die Kornvergrößlerung, Reifung von Halogensilber in Gelatineemulsion durch Elektrolyte kann durch Aneinanderlagerung mehrerer Halogensilberteilchen oder durch Umkristallisation der kleinen zu groberen Teilchen, wobei eine Lösung der kleinen Teilchen vorausgehen muß, erfolgen. In erstarrten Emulsionen ist nur der letztere Vorgang möglich, wail hier dic Beweglichkeit der Teilchen gehemmt ist. Setzt man einen Tropfen $\mathrm{Ca} \mathrm{Cl} \mathrm{C}_{2}$-Losung aut eine Chlor sllberemulsion, so ist unter dem Tropien die Reifung am stärksten und nimmt gleichmäbig bei Eindiffundieren des $\mathrm{CaCl}_{2}$ in die Schi ht, entsprechend dem Konzentrationsgefalle dieses Salzes, ab. Zwei aufgesetzte Tropfen beeinflussen einander auch bei Berührung der Diffusionskreise gar nicht. Diese Reifungserscheinungen sind aiso ganz anders als die Erscheinungen, die zu beobachten sind, wenn man $z$. B. Silbernitrattropfen auf eine Kaliumbichromatgelatine aufsetzt.

A. Lottermoser.

Liesegang, R. Ed., Zur Uebersättigungstheorie einiger scheinbar rhythmischen Reaktionen. (Zeitschrift f. physik. Chem. 75, 371373,1910 )

Wi. Ostwald batte die Ansicht ausgesprochen, daB die konzentrischen Ringsysteme vori ilberchironat, die beim Eindiffundieren von $\mathrm{AgNO}_{3}$ n eine Alinilichromatgelatine auftreten, durch Uebersăttigungserscheinungen verursacht würden. In der Tat mul man annehmen, daB nicht nut $\mathrm{AgNO}_{\mathfrak{g}}$ von d:n aufgeseizten Tropfen aus in die Gelatine zentrifugal eindiffundiert, sondern auch Chromat zentripedal voràringt, weil die Konzentration desselben durch die Wegnahme desselben durch das vordringende $A g \mathrm{NO}_{B}$ in der Umgebung des Troplens abnimmt. Mit dieser Theorie Wi. Ostwald's schien nun nicht libereinzustimmen die Tatsache, daB neuerlich auf ein solches Ringsystem aufgesetzte $\mathrm{AgNO}$-Tropfen neue Ringsysteme erzeugen, die von den alten fast unbeeinflußt bleiben. Das lâbt sich abes doch mit der Theorie vereinbaren, wenn man annimint, daB beide Ringsysteme in verschiedenen Fbenen der Gelatineschicht liegen. Bei ahnlichen Diffusionsversuchen konnte das der Verfasser direkt rachweisen.

\section{A. Luttermoser.}

Grenet und Salinbeni. Widerstandsfahigkeit von Filterkerzen mit Kollodiumaberzug gegen den Durchtritt von Mikrobt: (Compt. rend. 152, 916-.919, 1911.)

Chamberlandkerzen, die mit einetn Ueberzig von Kollodium versehen sind, sind fü Mikroben und für Kolloide voilkommen undurchlässig Gewisse Uebelstände, die bisher die Ve.wendung solcher Kollodiumiberzuge unsicher machen, wie Bruchigwerden des Kollodiums usw, we ter wirksam bekampft. Besonders Luftblass:hen in ler Kollodiumbaut sind sehr störend. Sie werden ve mieden, wenn die Kerze kurz vot dem Ueherwiehen mit Kollodium in Wasser uder noch besser in 30 proz. Alkohol getaucht wird. Das Kollodium erhält einen Zusatz von 8 bis 10 Proz. Glyzerin. Je hoher der Alkoholgehalt des Kollodiums ist, um so gröer ist tie Durchlassigkeit des Ueberzugs fur Wasser; je m tr Aether es enthielt, um so dichter und fur Waser weniger durchlassig ist es. Soll d e Kollodiumhaut abgelost werden, so läBt man dic $\mathrm{Ke}_{2} z \mathrm{e}$ trocknen; soll sie nach dem Filtrieren konservier $i$ werden, so taucht man die Kerze einige Augenblicke in 50 proz. Glyzerin ein. Zur Vermeidung von Schimmelbildung emptichlt stch ein 